\title{
Proton-Induced Reactions of Astrophysical Interest
}

\author{
Thomas Chillery $^{1, \star}$ for the LUNA Collaboration ${ }^{2}$ \\ ${ }^{1}$ SUPA, School of Physics and Astronomy, University of Edinburgh, Edinburgh, UK \\ ${ }^{2}$ http://luna.Ings.infn.it
}

\begin{abstract}
A discrepancy exists between the ${ }^{6} \mathrm{Li}$ abundances predicted from big bang nucleosynthesis models and those measured in pre-main sequence stars. To further constrain the predicted abundances of ${ }^{6} \mathrm{Li}$ in these stars, high accuracy measurements are required of reactions destroying ${ }^{6} \mathrm{Li}$. Namely ${ }^{6} \mathrm{Li}(\mathrm{p}, \gamma){ }^{7} \mathrm{Be}$ and ${ }^{6} \mathrm{Li}(\mathrm{p}, \alpha)^{3} \mathrm{He}$. These have recently been studied at the Laboratory for Underground Nuclear Astrophysics (LUNA) to measure their low energy cross sections. I present both the campaign's experimental setup and current status of the data analysis.
\end{abstract}

\section{Introduction}

Following the recent sucess of the Wilkinson Microwave Anisotropy Probe (WMAP) experiment in measuring the footprint of the cosmic microwave background (CMB) [1], cosmologists are closer than ever to precisely model and understand big bang nucleosynthesis (BBN) [2] in the early universe. Based on the current Standard Model, BBN aims to predict the abundances of light elements between one second and three minutes after the big bang. Of high interest to nuclear astrophysicists, the parameters obtained from BBN+WMAP data have been used to predict the present abundances of low mass isotopes found in stars. Whilst deuterium and ${ }^{4} \mathrm{He}$ abundances show excellent agreement between those predicted by BBN+WMAP and those measured in galaxies [2], large discrepancies are found for ${ }^{6} \mathrm{Li}$ and ${ }^{7} \mathrm{Li}$ between BBN+WMAP predictions and measurements of low-metallicity, or pre-main sequence (PMS), stars. A recent review [3] suggests the predicted ${ }^{7} \mathrm{Li}$ abundances are $2-4$ times higher than those measured, and inversely the predicted ${ }^{6} \mathrm{Li}$ abundances are 5000 times lower than measured. Whilst this large discrepancy is currently attributed to ${ }^{6} \mathrm{Li}$ production via proton induced spallation and fusion reactions, the environments encountered in PMS stars are expected to destroy more ${ }^{6} \mathrm{Li}$ than ${ }^{7} \mathrm{Li}$. To further constrain the predicted abundances of ${ }^{6} \mathrm{Li}$ in PMS stars, further high accuracy measurements are required of the reactions burning ${ }^{6} \mathrm{Li}$. Understanding the destructive processes of ${ }^{6} \mathrm{Li}$ to a higher accuracy will also enable theorists to better model the inner profile of PMS stars. This profile can provide an indication of how the convective currents vary beneath the stellar surface [4].

Two specific ${ }^{6} \mathrm{Li}$ destruction reactions, ${ }^{6} \mathrm{Li}(\mathrm{p}, \gamma){ }^{7} \mathrm{Be}$ and ${ }^{6} \mathrm{Li}(\mathrm{p}, \alpha)^{3} \mathrm{He}$, have been studied at the underground $400 \mathrm{kV}$ LUNA accelerator in Gran Sasso laboratory, Italy. The underground conditions lead to low background fluxes, thus experiments at energies of astrophysical interest are less challenging than similar studies performed on Earth's surface. Data collection was recently completed whilst

^e-mail: Thomas.Chillery@ed.ac.uk 
analysis is currently ongoing. Recent work by [5] shows a very distinctive drop in the ${ }^{6} \mathrm{Li}(\mathrm{p}, \gamma){ }^{7} \mathrm{Be}$ $\mathrm{S}$-factor for $\mathrm{E}_{\mathrm{cm}}$ approaching the Gamow energy $(\simeq 15 \mathrm{keV})$. An upwards trend at decreasing $\mathrm{E}_{\mathrm{cm}}$ would have been expected as suggested by earlier papers, see [6-9], and therefore [5] proposes a resonance exists with properties $\mathrm{E}_{\mathrm{R}} \simeq 195 \mathrm{keV}, \Gamma_{\mathrm{p}} \simeq 50 \mathrm{keV}$ to account for the drop. Whilst [5] does not quote whether their reported $S$-factor includes the electron screening effect or not (bare), the maximum possible enhancement may be calculated from [10]. At the lowest energy measured by [5], $\mathrm{E}_{\mathrm{cm}}=46.6 \mathrm{keV}$, this enhancement factor between bare and screened cross section is $3.1 \%$. This enhancement factor is smaller than the error in S-factor of $12.7 \%$ reported by [5], suggesting the electron screening effect is negligible in the current literature. Determining the ${ }^{6} \mathrm{Li}(\mathrm{p}, \gamma)^{7} \mathrm{Be} \mathrm{S}$-factor at $\mathrm{E}_{\mathrm{cm}}=64-338 \mathrm{keV}$, thus confirming or denying the proposed resonance, is the final objective of this ongoing experimental campaign at LUNA.

\section{Experimental Setup}

A schematic diagram of the LUNA $400 \mathrm{kV}$ accelerator solid target chamber used for the ${ }^{6} \mathrm{Li}$ runs is shown in figure 1. The accelerator was operated at energies $E_{l a b}=75-394 \mathrm{keV}\left(\mathrm{E}_{\mathrm{cm}}=64-338\right.$ $\mathrm{keV}$ ). The protons were focused on target using two circular apertures (diameters of $6 \mathrm{~mm}$ and $3 \mathrm{~mm}$ respectively) reducing the beamspot to a size comparable to the target (diameter $23 \mathrm{~mm}$ ). The protons are then sent through a copper tube, which was cooled using $\mathrm{LN}_{2}$ for carbon suppression and biased to $-300 \mathrm{~V}$ to suppress secondary electrons. The proton beam strikes the solid target mounted at $55^{\circ}$ to the beam axis. Alpha particles emitted during the experiment were detected using a silicon detector, mounted on an actuator for free movement inside the target chamber and facing the front of the target. The Si detector was collimated (collimator diameter $1 \mathrm{~mm}$ ) and had a mylar foil (thickness $5 \mu \mathrm{m}$ ) in front of it to attenuate the backscattered proton beam. Gamma rays emitted were detected by the HPGe, placed on top of an aluminium slide and facing perpendicular to the rear of the target holder. The signals aquired from both detectors were shaped with standard NIM electronics and aquired using MAESTRO [11].

The silicon detector was calibrated using both a fixed ${ }^{241} \mathrm{Am}$ source and the well-known $151 \mathrm{keV}$ resonance in the ${ }^{18} \mathrm{O}(\mathrm{p}, \alpha){ }^{15} \mathrm{~N}$ reaction [12]. The HPGe detector was calibrated using fixed sources $\left({ }^{137} \mathrm{Cs},{ }^{60} \mathrm{Co}\right.$, and $\left.{ }^{88} \mathrm{Y}\right)$ and the well-known $259 \mathrm{keV}$ resonance in the ${ }^{14} \mathrm{~N}(\mathrm{p}, \gamma){ }^{15} \mathrm{O}$ reaction [13]. Various ${ }^{6} \mathrm{Li}$-enriched targets were used during the two months of experiment; ${ }^{6} \mathrm{Li}_{2} \mathrm{O}$ with nominal thicknesses $20-40 \mu \mathrm{g} / \mathrm{cm}^{2},{ }^{6} \mathrm{LiWO}_{4}$ with nominal thicknesses $100-130 \mu \mathrm{g} / \mathrm{cm}^{2}$, and an "infinitely thick" $\left(>450 \mu \mathrm{g} / \mathrm{cm}^{2}\right) \mathrm{LiCl}$ target. The oxide and tungstate targets were evaporated on tantalum backings, while the $\mathrm{LiCl}$ has a $\mathrm{Cu}$ backing. The targets were water cooled during beam bombardment.

\section{Preliminary Results}

To calibrate the setup we measured the fixed sources, ${ }^{14} \mathrm{~N}(\mathrm{p}, \gamma){ }^{15} \mathrm{O}$, and ${ }^{18} \mathrm{O}(\mathrm{p}, \alpha){ }^{15} \mathrm{~N}$ reactions mentioned in section 2. The HPGe efficiency curves for a range of distances, $\mathrm{d}=4-254 \mathrm{~mm}$, is presented in figure 2, where the distance is measured between the front cap of the Germanium and the back side of the target holder. The efficiency errors plotted are statistical, of order $1.5 \%$. Systematic uncertainties arising from correct positioning of the HPGe detector are estimated to be no greater than $5 \%$. The efficiency curves do not include true summing corrections. A sample spectrum obtained from protons of $\mathrm{E}_{\mathrm{p}}=230 \mathrm{keV}$ incident on a $40 \mu \mathrm{g} / \mathrm{cm}^{2}{ }^{6} \mathrm{Li}_{2} \mathrm{O}_{4}$ target is shown in figure 2. The direct capture peaks for the decay of the compound nucleus to the ground state, $\gamma_{1}$, and to the $429 \mathrm{keV}$ excited state, $\gamma_{2}$, are labelled. The first and second escape peaks are also observed. A beam induced reaction caused by contaminants in the target chamber, ${ }^{19} \mathrm{~F}(\mathrm{p}, \alpha \gamma){ }^{16} \mathrm{O}$, was also monitored online and is shown in the same figure. 


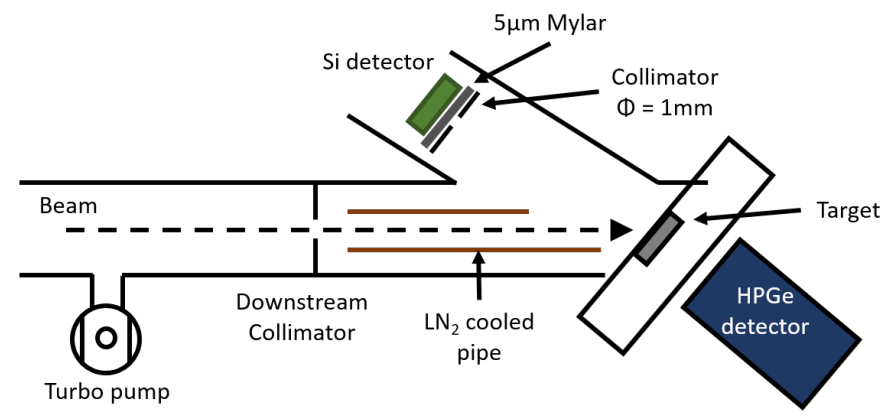

Figure 1: A schematic diagram of the target chamber, with Silicon and Germanium detector positions shown.
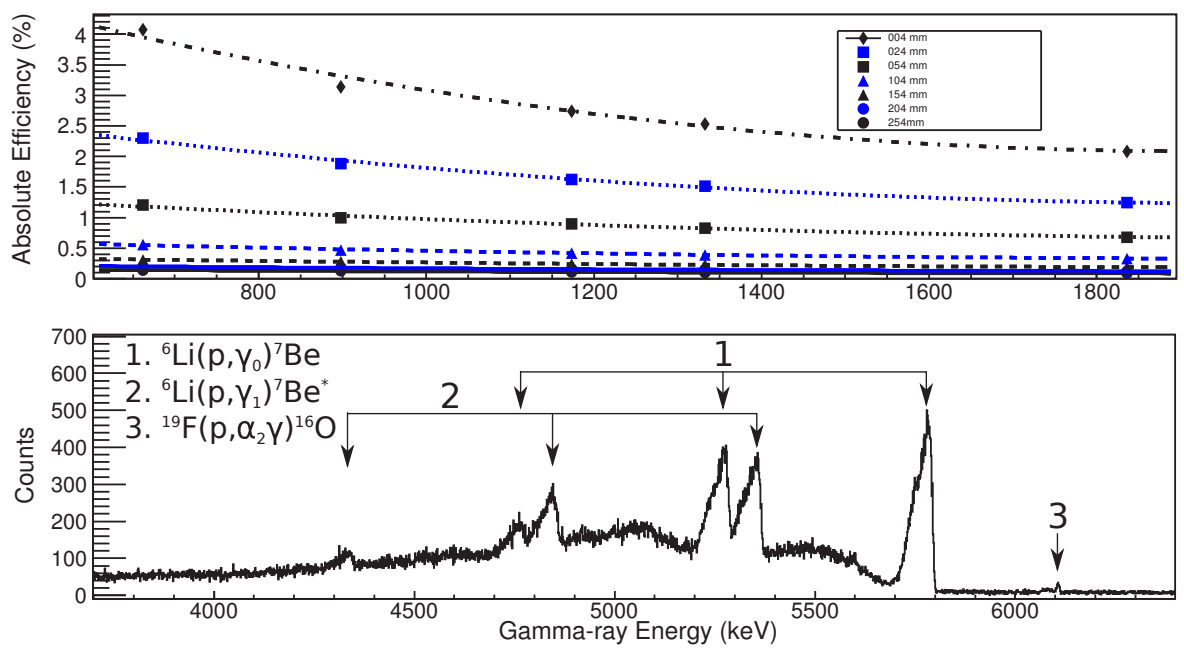

Figure 2: Top: Preliminary HPGe Efficiency Curves. Errors plotted are statistical (1.5\%) but smaller than data point sizes. Bottom: Gamma spectrum for protons of $\mathrm{E}_{\mathrm{p}}=230 \mathrm{keV}$ on ${ }^{6} \mathrm{Li}_{2} \mathrm{O}_{4}$ target.

\section{Summary}

During 2017 an experimental campaign was recently completed studying two destructive reactions; ${ }^{6} \mathrm{Li}(\mathrm{p}, \gamma){ }^{7} \mathrm{Be}$ and ${ }^{6} \mathrm{Li}(\mathrm{p}, \alpha){ }^{3} \mathrm{He}$, in the LUNA laboratory. Multiple ${ }^{6} \mathrm{Li}$-enriched solid targets were irradiated using a proton beam at $\mathrm{E}_{\mathrm{cm}}=64-338 \mathrm{keV}$. Efficiency curves for both the HPGe and $\mathrm{Si}$ detectors have been obtained using both fixed sources and proton beam-induced reactions. Gamma detection efficiency errors are $1.5 \%$ statistical, $5 \%$ systematic. True summing corrections for the HPGe efficiency, including the use of Geant 4 simulations, are currently ongoing. Efforts to extract the $\mathrm{S}$-factor from the beam studies for both destructive reactions is also ongoing. 


\section{References}

[1] C. Bennett et al., The Astrophysical Journal Supplement Series 208, 20 (2013)

[2] B. Fields, P. Molaro, S. Sarkar, Chinese Physics C pp. 1-15 (2014)

[3] B. Fields, Annual Review of Nuclear and Particle Science 61, 47 (2011)

[4] F. Thévenin et al., Astronomy \& Astrophysics 598, A64 (2017)

[5] J. He et al., Physics Letters B 725, 287 (2013)

[6] Z. Switkowski et al., Nuclear Physics A 331, 50 (1979)

[7] F. Barker, Australian Journal of Physics 33, 159 (1980)

[8] K. Arai, D. Baye, P. Descouvemont, Nuclear Physics A 699, 963 (2002)

[9] J. Huang, C. Bertulani, V. Guimarães, Atomic Data and Nuclear Data Tables 96, 824 (2010)

[10] H. Assenbaum, K. Langanke, C. Rolfs, Z. Phys. A. Atomic Nuclei 327, 461 (1987)

[11] Maestro multichannel analyser emulation, http://www.ortec-online.com/products/ application-software/maestro-mca

[12] C. Bruno et al., Eur. Phys. J. A 51, 94 (2015)

[13] G. Imbriani et al., Eur. Phys. J. A 25, 455 (2005) 